\title{
Lentiviral vector mediated siRNA knock-down of hTERT results in diminished capacity in invasiveness and in vivo growth of human glioma cells in a telomere length-independent manner
}

\author{
PENG ZHAO $^{1 *}$, CUNZU WANG $^{1 *}$, ZHEN FU $^{1}$, YONGPING YOU ${ }^{1}$, YUNXIANG CHENG $^{1}$, XIAOMING LU $^{1}$, \\ AILIN LU ${ }^{1}$, NING LIU ${ }^{1}$, PEIYU PU ${ }^{2}$, CHUNSHENG KANG ${ }^{2}$, LEIF G. SALFORD ${ }^{3}$ and XIAOLONG FAN ${ }^{3}$ \\ ${ }^{1}$ Department of Neurosurgery, The First Affiliated Hospital of Nanjing Medical University, Nanjing 210029; \\ ${ }^{2}$ Neuro-oncology Lab of Tianjin Neurological Institute, Department of Neurosurgery, General Hospital, \\ Tianjin Medical University, Tianjin 300052, P.R. China; ${ }^{3}$ The Rausing Laboratory, \\ Division of Neurosurgery, Lund University Hospital, Lund, Sweden
}

Received March 12, 2007; Accepted April 27, 2007

\begin{abstract}
Glioma cells are characterized by their invasiveness and resistance against conventional therapeutics. Telomerase activity has been suggested to be an important target for glioma treatment. Here we assessed the anticancer effects and its potential mechanisms of lentiviral vector mediated siRNA knock-down of the human telomerase reverse transcriptase (hTERT) in U87MG human glioblastoma cells. Stable expression of anti-hTERT siRNA reduced the hTERT expression and TRAP assay telomerase activity to barely detectable levels. Injection of lentiviral vectors encoding anti-hTERT siRNA significantly inhibited the growth of preestablished macroscopic xenograft tumors, which was in contrast to the finding that no obvious effects on cell growth, cell cycle progression and telomere length were observed in anti-hTERT siRNA expressing U87MG cells during short-term in vitro cultures. The in vivo glioma growth inhibition effect was already evident in the period coincided with no detectable telomere length changes, suggesting that hTERT inhibition may hinder glioma cell growth in a telomere length-independent manner. Importantly, transwell migration assay showed profound inhibitory effect on the invasive capacity of U87MG cells following short-term anti-hTERT
\end{abstract}

Correspondence to: Dr Yongping You, Department of Neurosurgery, The First Affiliated Hospital of Nanjing Medical University, Nanjing 210029, P.R. China

E-mail: YYPL3@sohu.com

Dr Xiaolong Fan, The Rausing Laboratory, Division of Neurosurgery, Lund University Hospital, Lund, Sweden

E-mail: xiaolong.fan@med.lu.se

${ }^{*}$ Contributed equally

Key words: siRNA, hTERT, glioma, telomerase activity, gene therapy
siRNA expression. Thus, efficient knock-down of hTERT can inhibit glioma cell proliferation and migration prior to its effect on telomere length.

\section{Introduction}

Gliomas are the most aggressive type of primary intracranial tumors and constitute almost $80 \%$ of primary brain tumors. Patients suffering from malignant gliomas have a life-span between 9 and 12 months after diagnosis. Presently, the conventional treatments for gliomas include surgery, radiation therapy, and chemotherapy. Although a great deal of effort has been directed toward the therapy, the prognosis of gliomas, in particular the high-grade malignant gliomas, has not achieved any marked improvement. The main reason for the poor prognosis is due to the fact that glioma cells migrate across the rim of tumors and infiltrate 'normal brain tissues', and the conventional therapies cannot selectively kill these invasive glioma cells. Therefore, there is an urgent need to develop new therapeutic strategies for malignant gliomas $(1,2)$.

Similar to other tumors, gliomas are caused by mutations that activate oncogenes and/or switch off tumor suppressor genes. Overcoming telomere-dependent replication senescence can also be a critical step in glioma development. Telomerase is a ribonucleoprotein enzyme complex, whose RNA component (hTR) is used as a template by the human telomerase reverse transcriptase (hTERT) for synthesizing the telomeric repeat sequences (TTAGGG)n. This lengthens telomere and critically contributes to cellular immortality. Telomerase is expressed in more than $90 \%$ of advanced cancers but is not found in most normal cells (3-5). Previous studies have shown that inhibition of telomerase activity or downregulation of telomerase expression can inhibit glioma cell proliferation $(6,7)$.

It has been demonstrated that telomerase activity is predominantly regulated by hTERT expression (8) and the expression of hTERT is elevated in high-grade gliomas compared to in low-grade gliomas $(9,10)$. Thus, hTERT likely plays an important role in the development and progression of gliomas, and may also be a potential target for human 


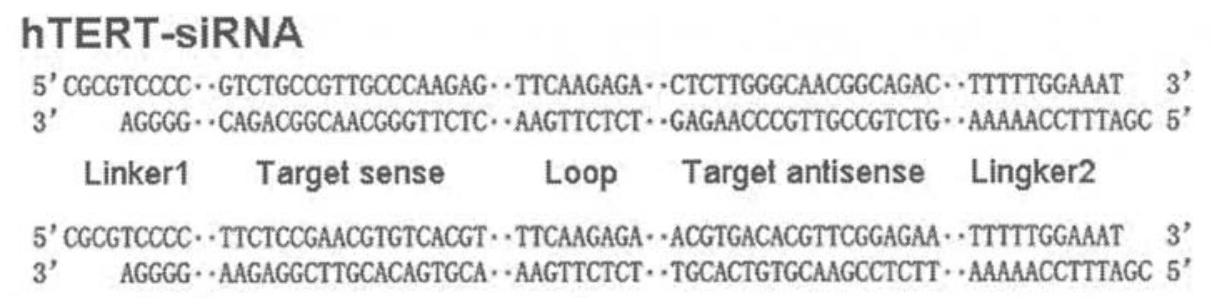

\section{hTERT-NC}

\section{Ligation}

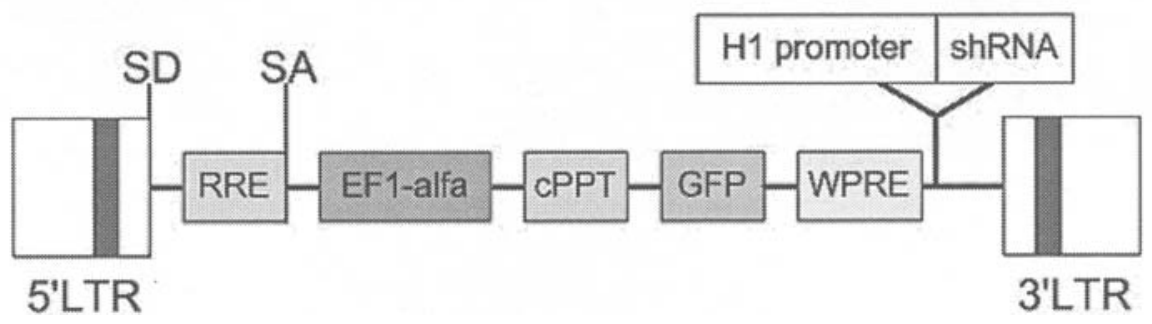

Figure 1. Construction of the pLVTHM vector encoding anti-hTERT siRNA. For the hTERT-siRNA and the hTERT-NC vector, two single strand DNA encoding two linkers, the target sequences and a loop element were synthesized (Invitrogen). These were annealed to double strand DNA, and ligated into the pLVTHM following MluI and CalI digestion. The vectors were generated by transient transfection of pCMV-dR8.74, pMD2G and the siRNA encoding pLVTHM into 293T cells as described in Materials and methods.

malignant glioma treatment using gene therapy approach. The current study was designed with the aim to investigate the effects of small interfering RNA (siRNA) directed against hTERT (hTERT-siRNA) on glioma cell growth and its invasive capacity. We used VSV-G pseudotyped lentiviral vector mediated delivery of hTERT-siRNA to downregulate hTERT expression in human glioblastoma cell line U87MG. We found that the hTERT-siRNA markedly reduced the hTERT expression and telomerase activity. Functionally, reduced hTERT expression resulted in impaired invasive capacity and inhibited the growth of pre-established macroscopic xenograft gliomas in a telomere length-independent manner.

\section{Materials and methods}

Engineering of lentiviral vector based siRNA construct against hTERT. The lentivirus system consisting of pLVTHM, pCMV$\mathrm{dR} 8.74$ and pMD2G was obtained from Trono Laboratory (University of Geneva, Switzerland) (11). The pLVTHM vector contains an $\mathrm{H} 1$ promoter controlled expression cassette for short hairpin RNAs (shRNAs) and an EF1 promoter controlled GFP expression cassette. The pCMVdR8.74 encodes the regulatory DNA sequences for vector generation and the pMD2G encodes the VSV-G gene. A 19-bp fragment within the hTERT cDNA was chosen as the target for our siRNA (hTERT-siRNA): 5'-GTCTGCCGTTG CCCAAGAG-3' at position 569-588 (Genbank accession no. NM_003219). The expression level of hTERT mRNA in transfected 293T cells was found to be $22.7 \%$ of the level in non-transfected cells. Lentiviral vector carrying a scrambled fragment (5'-TTCTCCGAACGTGTCACGT-3') as a negative control (NC) was also generated, which we named hTERT-NC (Fig. 1). The hTERT-siRNA and the hTERT-NC vector were generated in 293T cells upon transfection of pCMV-dR8.74, pMD2G and shRNA encoding pLVTHM using Lipofectamine 2000 (Invitrogen, USA), according to the manufacturer's instructions. Forty-eight hours post transfection, the viral supernatant was collected and centrifuged at 25,000 rpm, $4^{\circ} \mathrm{C}$, for $90 \mathrm{~min}$, and the pellet was suspended in PBS and stored at $-70^{\circ} \mathrm{C}$. Viral titers were determined as transduction unit (TU) by infection of $293 \mathrm{~T}$ cells with serial dilutions of vector preparation and microscopic counting of GFP expressing colonies as described previously (12).

Cell culture and lentiviral vector infection. The U87MG human glioma cell line (kindly provided by Dr Jianhong Zhu, Neurosurgical Department of Huashan Hospital, Shanghai, P.R. China) was maintained in Dulbecco's modified Eagle's medium (DMEM) supplemented with $10 \%$ fetal calf serum and antibiotics (penicillin at $100 \mathrm{U} / \mathrm{ml}$ and streptomycin at $100 \mu \mathrm{g} / \mathrm{ml}$ ) in a humidified atmosphere of $5 \%$ carbon dioxide and $95 \%$ air at $37^{\circ} \mathrm{C}$. Viral stocks were diluted in serum-free medium to obtain the desired multiplicities of infection (MOI) and added to cell monolayer and incubated at $37^{\circ} \mathrm{C}$ for $1 \mathrm{~h}$. The culture medium with $10 \%$ fetal calf serum was then added, and the cells were incubated for desired durations. U87MG cells were infected at various MOI and the infection-efficiency curve at $72 \mathrm{~h}$ was determined by counting GFP positive cells. In general, the cell infection was dose-dependent in the range of 25-200 MOI. More than $80 \%$ of infected cells were obtained at 50 MOI for U87MG cells after $72 \mathrm{~h}$; so cells were infected at $50 \mathrm{MOI}$ in studies described in Figs. 2-6.

Detection of hTERT mRNA expression by reverse transcriptionpolymerase chain reaction ( $R T-P C R)$. Total RNA was isolated from cells infected with the hTERT-siRNA or the hTERT-NC vector or from uninfected cells, using an RNA Isolation Kit (Qiagen, USA), and converted to cDNA with random primer by ExScript $^{\mathrm{TM}}$ RT reagent Kit (Takara). PCR reactions were carried out using Ex Taq hot start polymerase (Takara). GAPDH was used a control for adjusting the relative amounts 
of total RNA between the samples. The thermal cycles were: $94^{\circ} \mathrm{C}$ for $1 \mathrm{~min}, 60^{\circ} \mathrm{C}$ for $1 \mathrm{~min}$, and $72^{\circ} \mathrm{C}$ for $1 \mathrm{~min}$ for 30 cycles for GAPDH (450 bp) and $94^{\circ} \mathrm{C}$ for $1 \mathrm{~min}, 58^{\circ} \mathrm{C}$ for $1 \mathrm{~min}$, and $72^{\circ} \mathrm{C}$ for $1 \mathrm{~min}$ for 36 cycles for hTERT (145 bp). The primers (Invitrogen, Shanghai, P.R. China) used for PCR were as follows: GAPDH, 5'-CTCAGACACCATGGGGAA GGTGA-3' (forward) and 5'-ATGATCTTGAGGCTGTTGT CATA-3' (reverse); hTERT, 5'-CGGAAGAGTGTCTGGAG CAA-3' (forward) and 5'-GGATGAAGCGGAGTCTGGA-3' (reverse) (13). The PCR reactions for GAPDH and hTERT were mixed in a ration of $1: 1$ and fractionated on a $3 \%$ agarose gel containing $0.5 \mathrm{mg} / \mathrm{ml}$ ethidium bromide. Gels were visualized by Gel Doc ${ }^{\mathrm{TM}} \mathrm{XR}$ gel documentation system (Bio-Rad).

Telomerase activity assay. Telomerase activity was measured by polymerase chain reaction (PCR)-based telomeric repeat amplification protocol (TRAP) assay (14) by means of the TRAPeze Telomerase Detection Kit (Chemicon). The enzyme activity products were amplified by PCR and resolved on $10 \%$ polyacrylamide gel. Cellular protein extracts were also incubated at $85^{\circ} \mathrm{C}$ for $10 \mathrm{~min}$ to test their heat sensitivity. A TSR8 PCR positive control and a primer-dimer/PCR contamination control were included in each set of TRAP assays.

Measurement of cell growth. The effect of hTERT-siRNA on U87MG cell growth was determined by MTT assay using the Cell Titer 96 Aqueous One Solution Cell Proliferation Assay Kit (Promega), according to the manufacturer's instructions. The absorbance at $490 \mathrm{~nm}$ was measured with a 96-well Opsys MR $^{\text {тм }}$ Microplate Reader (ThermoLabsystems, Chantily,
1

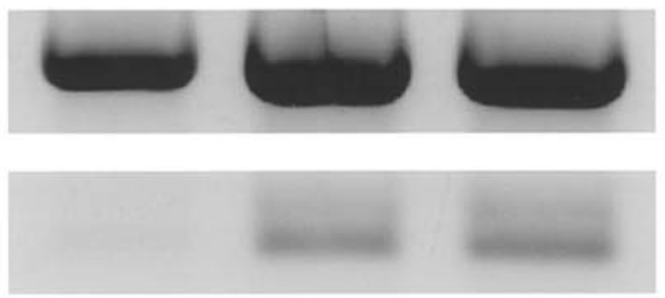

GAPDH

\section{In vitro}

1 2 3

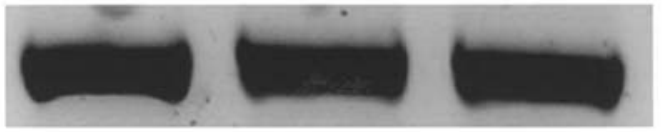

GAPDH

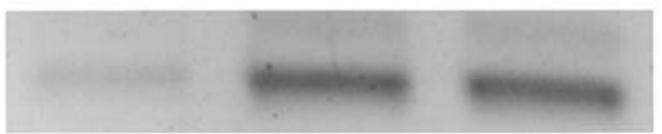

hTERT

\section{In vivo}

Figure 2. Detection of hTERT mRNA expression following infection with siRNA encoding lentiviral vectors in human glioma cells in vitro and in vivo by RT-PCR. U87MG cells were infected in vitro or injected in vivo with the hTERT-siRNA (U87-I) or the hTERT-NC (U87-E) vector, or left uninfected as controls (U87). Total cellular RNA was isolated and assessed for the mRNA expression of hTERT and GAPDH genes. In vitro: lane 1, U87-I; lane 2, U87-E; lane 3, U87. In vivo: lane 1, U87-I; lane 2, U87-E; lane 3, U87.

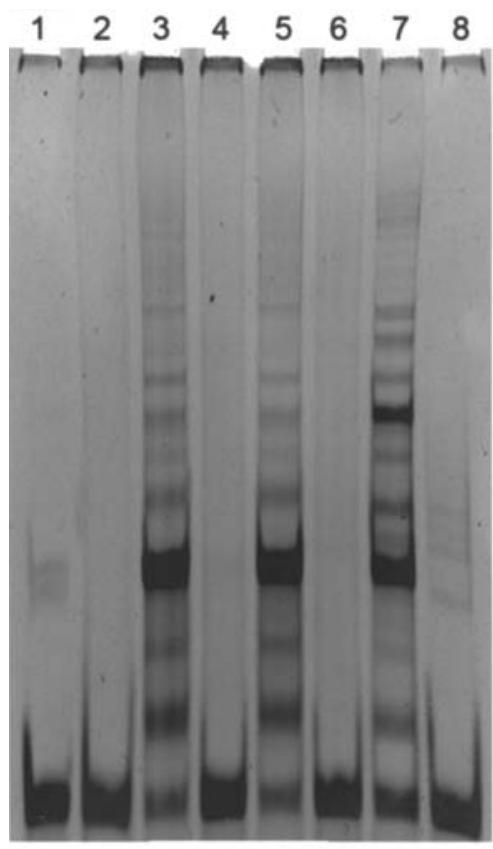

In vitro

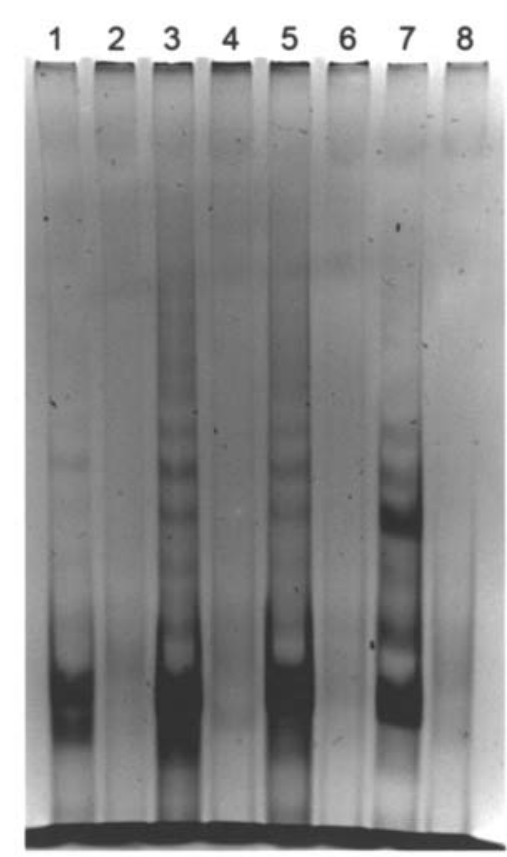

In vivo

Figure 3. Assessment of the effect of hTERT-siRNA on telomerase activity in human glioma cells in vitro and in vivo by TRAP assay. As in Fig. 2, U87MG cells were infected in vitro or injected in vivo with either the hTERT-siRNA (U87-I) or the hTERT-NC (U87-E) vector, or left untreated as controls (U87). Telomerase activity was measured by TRAP assay. For both in vitro and in vivo data: lane 1, U87-I; lane 2, U87-I heat-inactivated; lane 3, U87-E; lane 4, U87-E heatinactivated; lane 5, U87; lane 6, U87 heat-inactivated; lane 7, TSR8 PCR positive control; lane 8, PCR contamination control. 
VA, USA) and analyzed with Revelation ${ }^{\mathrm{TM}}$ QuickLink Software. All experiments were performed at least three times with representative data presented.

Flow cytometric cell cycle analysis. Cell cycle analysis was performed on day 7 after lentiviral vector infection. Cells were fixed with $70 \%$ ethanol and incubated with ribonuclease A (250 mg/ml, Sigma) for $1 \mathrm{~h}$ at room temperature and propidium iodide $(50 \mathrm{mg} / \mathrm{ml}$, Sigma) for $10 \mathrm{~min}$. Each sample was filtered through a $50-\mu \mathrm{m}$ nylon mesh to obtain single-cell suspension. The samples were then analyzed on FACSCalibur (Becton Dickinson, Mountain View, CA, USA) with a minimum of 20,000 events recorded. ModFit LT software (Becton Dickinson) was used for data analysis. Mean peak fluorescence was determined for each histogram. Three separate experiments were performed.

Telomere length analysis by fluorescence in situ hybridization. On day 7 after lentiviral vector infection, we hybridized a $\mathrm{Cy}_{3}$ labeled peptide nucleic acid (PNA) probe (CCCTAA) specific for mammalian telomeres (Applied Biosystems, USA)

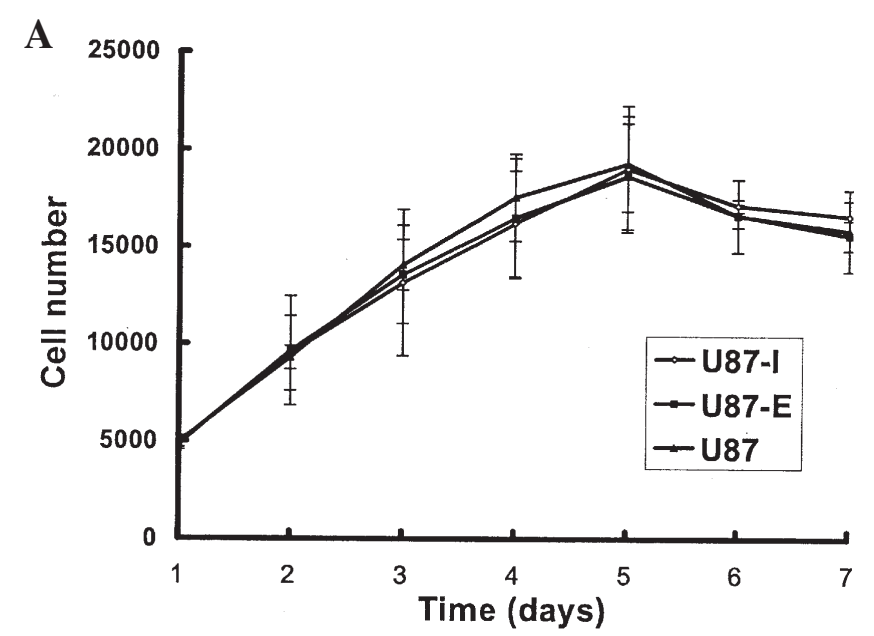

B

a

b to the cells fixed with methanol: acetic acid (3:1), at $72^{\circ} \mathrm{C}$ for $8 \mathrm{~min}$. To remove non-hybridized PNA probes, slides were washed with PBS containing $0.05 \%$ Tween- 20 at $56^{\circ} \mathrm{C}$ for $20 \mathrm{~min}$, counterstained with DAPI $(60 \mathrm{ng} / \mathrm{ml})$ for $5 \mathrm{~min}$ and visualized using an Olympus BX61 fluorescence microscope. In addition, to obtain more precise telomere signals, we performed Z-Stack ${ }^{\mathrm{TM}}$ by SPOT-AX system, which detects probe signals in different focal planes and then combines them into a single 2-D projection. The data were analyzed by CytoVision 3.6 and this assessment is only performed in GFP expressing cells.

Cell invasion assay. In vitro invasive capacity was evaluated using CytoSelect ${ }^{\mathrm{TM}}$ 24-well Cell Invasion Assay Kit (Cellbiolabs, USA). The basement membrane layer of the cell culture inserts was rehydrated with $300 \mu 1$ warm $\left(37^{\circ} \mathrm{C}\right)$, serum-free media for $1 \mathrm{~h}$. Seventy-two hours post infection, the cells were harvested and resuspended in serum-free DMEM. The upper chambers of the inserts were filled with $300 \mu \mathrm{l}$ cell suspension at a cell density of $5 \times 10^{6}$ cells/ml. DMEM (500 $\left.\mu \mathrm{l}\right)$ containing $10 \%$ fetal bovine sera was placed in the lower chambers as chemoattractant. The chambers were incubated at $37^{\circ} \mathrm{C}$ in a humidified atmosphere of $5 \%$ carbon dioxide for $48 \mathrm{~h}$. Cells that did not migrate through the pores were removed by scraping the membrane with a cotton swab, and those passing through the membrane were fixed in $70 \%$ ethanol and stained with hematoxylin and eosin. After microscopic documentation, hematoxylin and eosin stained cells were extracted and measured for optical density at 560 $\mathrm{nm}$ in a microplate reader. Three separate experiments were performed.

Subcutaneous tumor xenografts and assessment of cell growth in vivo. Subcutaneous xenograft model was chosen for feasible follow-up of tumor growth. A total of $1 \times 10^{7}$ U87MG cells suspended in serum-free DMEM were transplanted sub
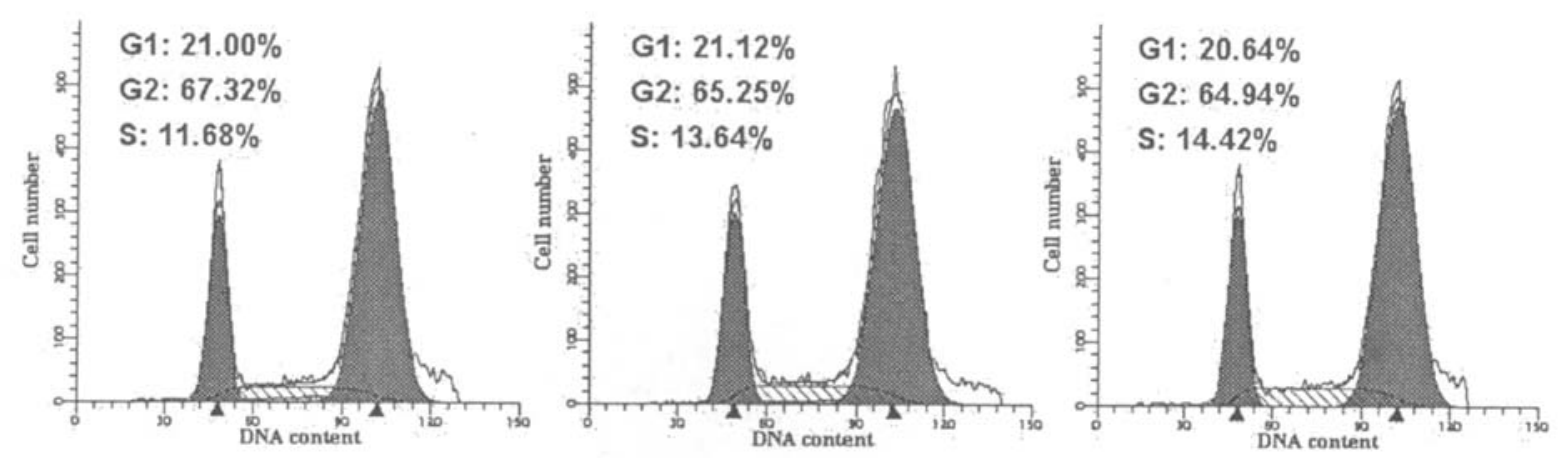

Figure 4. The effect of hTERT-siRNA on cell growth in human U87MG cells in vitro as determined by MTT assay and DNA flow cytometric analysis. (A) Growth curves for U87MG cells. Cells were plated at a cell density of 5x10 cells per well in 96-well plates and infected either with the hTERT-siRNA (U87-I) or the hTERT-NC (U87-E) vector, or left uninfected (U87). The data were averaged from three wells and expressed as mean \pm SD of cell numbers per well. (B) Effects of the hTERT-siRNA and the hTERT-NC vector infection on cell cycle progression. U87MG cells were infected with the hTERT-siRNA (a), the hTERT-NC vector (b) or left uninfected (c). Seven days after the infection, cells were stained with propidium iodide and analyzed in the FACSCalibur. The percentages of cells in different cell cycle phases were determined using ModFit LT software. The data shown are representative of three independent experiments. 
cutaneously into $24 \mathrm{BALB} / \mathrm{c}-\mathrm{A}$ nude mice (4 weeks old, SLAC laboratory animal Co. Ltd., Shanghai, P.R. China). When the tumors were $5 \mathrm{~mm}$ in diameter, the mice were divided into three groups with 8 animals in each, injected with $5 \times 10^{7} \mathrm{TU}$ of either the hTERT-siRNA (U87-I) or the hTERT-NC (U87-E) vector in $10 \mu 1$ PBS, or $10 \mu 1$ PBS (control U87 group). The length and width of the tumors were measured every third day, and the tumor mass was calculated using the equation $0.5 \mathrm{x}$ length $\mathrm{x}$ width ${ }^{2}(15)$. All measurements were performed in a coded, blinded fashion. Mice were sacrificed 27 days later, and tumors were dissected to determine telomerase activities by TRAP assay and to examine the expression of hTERT mRNA by RT-PCR analysis. The Ethics Committee of the Nanjing Medical University, China has approved the animal experiments for this study.

Statistical analysis. Data were analyzed with Stata 7.0. The mean differences among the three groups were tested using ANOVA, followed by multiple comparisons with the Scheffe method. Statistical significance was assigned to $\mathrm{P}<0.05$.

\section{Results}

Lentiviral vector mediated delivery of hTERT-siRNA inhibits hTERT expression and telomerase activitiy in vitro and in vivo. The pLVTHM vector allows stable transduction of shRNAs that are then processed to siRNA in mammalian cells. Oligonucleotides encoding shRNAs complementary to hTERT were cloned into pLVTHM. Three days after vector infection, cells were collected and examined for hTERT mRNA expression and telomerase activity. As shown in Fig. 2 upper part, hTERT mRNA expression diminished to barely detectable levels in the U87MG cells infected with the hTERT-siRNA vector, compared to the cells infected with the control hTERT-NC vector or the uninfected control cells. Similar data were achieved in U87MG cell xenograft gliomas in mice following injection with the hTERT-siRNA vector (Fig. 2 lower part). Accordingly, reduction of hTERT mRNA expression resulted in severely diminished TRAP assay telomerase activity (Fig. 3).

Effects of hTERT downregulation on short-term in vitro growth of U87MG cells. As shown in Fig. 4A, a 7-day short-term in vitro culture showed no measurable differences on the growth rate between the U87MG cells infected with the hTERT-siRNA, the hTERT-NC vector or the uninfected U87MG cells. To determine if downregulation of hTERT affects cell cycle of malignant glioma cells, the flow cytometric analysis for cellular DNA content was performed on day 7 after infection. As shown in Fig. 4B, the G2/M phase percentages in the parental U87MG cells and the cells infected with the hTERT-NC vector were 64.9 and $65.3 \%$, respectively, while the hTERT-siRNA vector infected cells contained $67.3 \%$ cells in G2/M phase. The S-phase fraction in parental U87MG cells and cells infected with the hTERT-NC vector was 14.4 and $13.6 \%$, respectively, but in the hTERT-siRNA vector infected cells the value decreased slightly to $11.7 \%$. No significant differences were observed in the proportion of G0/G1 fractions among the three groups and a sub-G0/G1 fraction was not detected, indicating that apoptosis did not occur within the time frame. These results suggest that under our short-term in vitro conditions, siRNA mediated downregulation of hTERT did not induce obvious cell cycle progression arrest or cell proliferation inhibition.

Effects of hTERT downregulation on telomere length of U87MG cells. For telomere specific FISH, we used $\mathrm{Cy}_{3}$ directly labeled PNA probes that can hybridize to complementary telomere repeats under conditions of low ionic strength, favoring annealing of the probes but disfavoring renaturation of DNA strands (16). Therefore, the fluorescence intensity of a given telomere spot is positively correlated with its corresponding repeat lengths. With the image analysis software CytoVision 3.6, the integrated fluorescence intensity values for each cell can be calculated. In the hTERT-siRNA and the hTERT-NC vector infected groups, only the cells that expressed GFP were counted. For each group, at least 100 cells were analyzed (Fig. 5), the average fluorescence intensity of telomere signals of U87MG cells infected with the hTERT-siRNA, the hTERT-NC vector and the uninfected cells were 27.6 \pm 14.4 , $27.9 \pm 13.9,29.2 \pm 14.6$, respectively, and there was no significant difference between them $(\mathrm{P}>0.05)$.

Effects of hTERT downregulation on the invasive capacity of U87MG cells. As cell invasiveness is an important feature of glioma cells, the potential effect of hTERT-siRNA on the invasive capacity of U87MG cells was determined. The transwell membrane was coated with a uniform layer of basement membrane matrix solution that forms a barrier similar to the mammalian basement membrane, thus this system enables the study of cell invasive capacity in vitro. The cells that migrated through the transwell membrane were first stained and then photographed. Subsequently, migrated cells were extracted and OD560 determined for quantification. The OD values were $0.46 \pm 0.01,0.83 \pm 0.07$ and $0.83 \pm 0.05$ for the hTERTSiRNA, the hTERT-NC vector infected cells and the control U87MG cells, respectively (Fig. 6). As the OD560 value is positively correlated to the cell number, the results indicated that the number of the cells invading through transwell membrane was significantly reduced in hTERT-siRNA group compared with that in the hTERT-NC vector infected or the non-infection group $(\mathrm{P}<0.05)$, and there was no significant difference between the hTERT-NC vector infected and the uninfected U87MG cells ( $\mathrm{P}>0.05)$. Thus, downregulation of hTERT expression resulted in a dramatically reduced invasive capacity of U87MG cells.

Effect of hTERT-siRNA on U87MG cell tumor growth in vivo. To study the potential effects of lentiviral vector delivered hTERT-siRNA on glioma cell growth in vivo, the hTERTsiRNA and the hTERT-NC vector preparation were injected into subcutaneously established U87MG cell xenograft gliomas in immunodeficient mice. As shown in Fig. 7, the tumors injected with the hTERT-siRNA vector grew significantly slowly, compared with the tumors injected with the hTERT-NC vector or PBS controls. There was no detectable difference in the growth profiles between the tumors injected with the control hTERT-NC vector and the tumors injected with PBS, suggesting that the in vivo glioma cell growth inhibition effect 
Telo-FISH

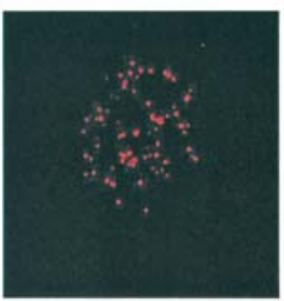

U87-I

U87-E

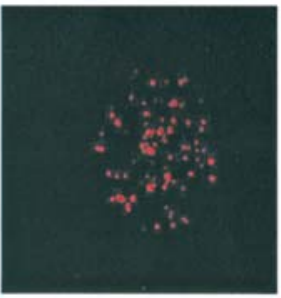

U87

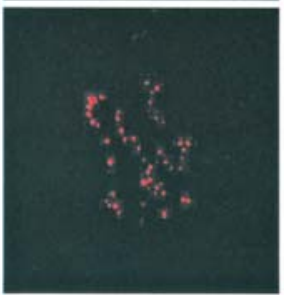

DAPI
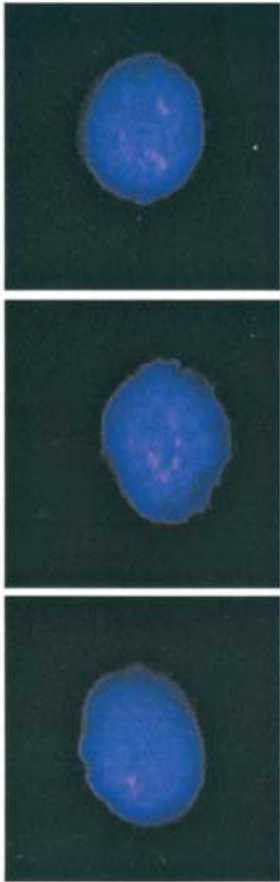

GFP
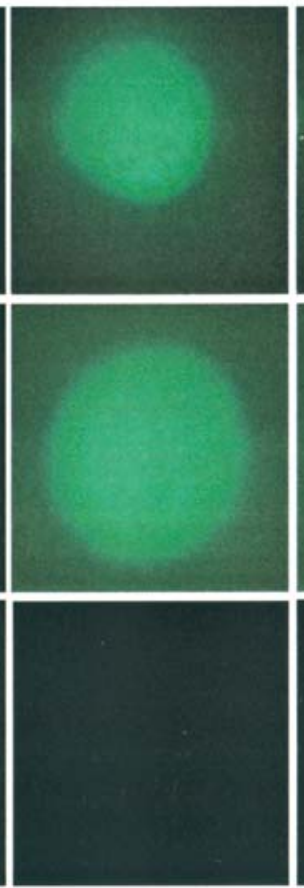

Merge
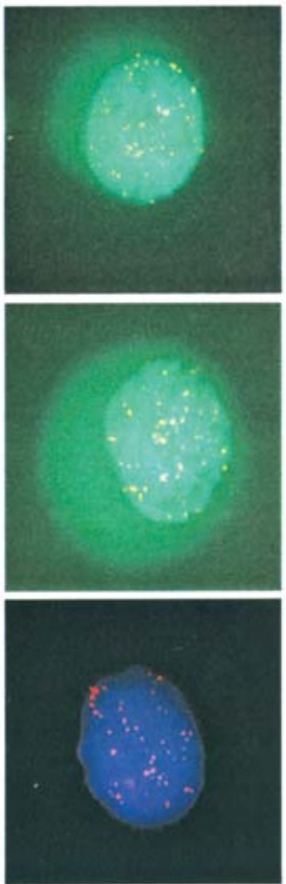

Figure 5. Telomere length of U87MG cells after infection with the hTERT-siRNA vector. Seven days after infection with either the hTERT-siRNA (U87-I) or the hTERT-NC (U87-E) vector, cells were fixed and hybridized with $\mathrm{Cy}_{3}$ directly labeled telomere-specific peptide nucleic acid probe (red) and counterstained with DAPI (blue). The vectors also encoded GFP, so the cells successfully infected with the vectors were GFP positive, and uninfected cells were negative (magnification x1000). The average fluorescence intensity of telomere signals of the U87MG cells infected with the hTERT-siRNA (U87-I), or the hTERT-NC vector (U87-E) and of the uninfected cells (U-87) were 27.6 $\pm 14.4,27.9 \pm 13.9,29.2 \pm 14.6$, respectively. There was no significant difference between the cells from the 3 groups $(\mathrm{P}>0.05)$.

A

a

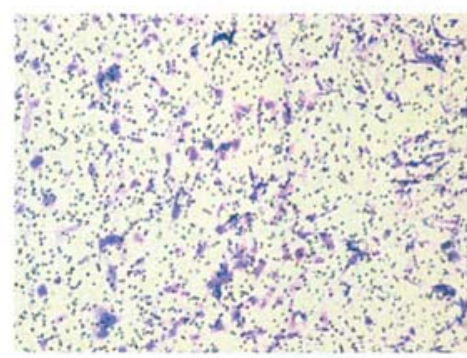

b

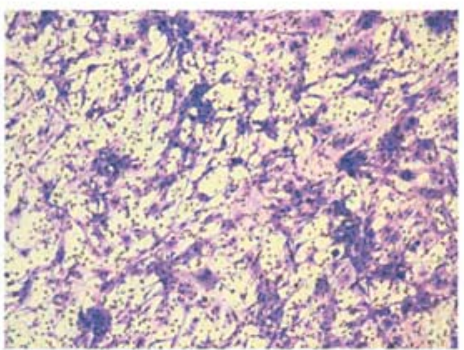

C

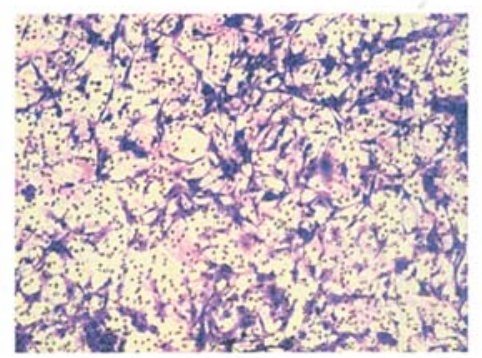

B

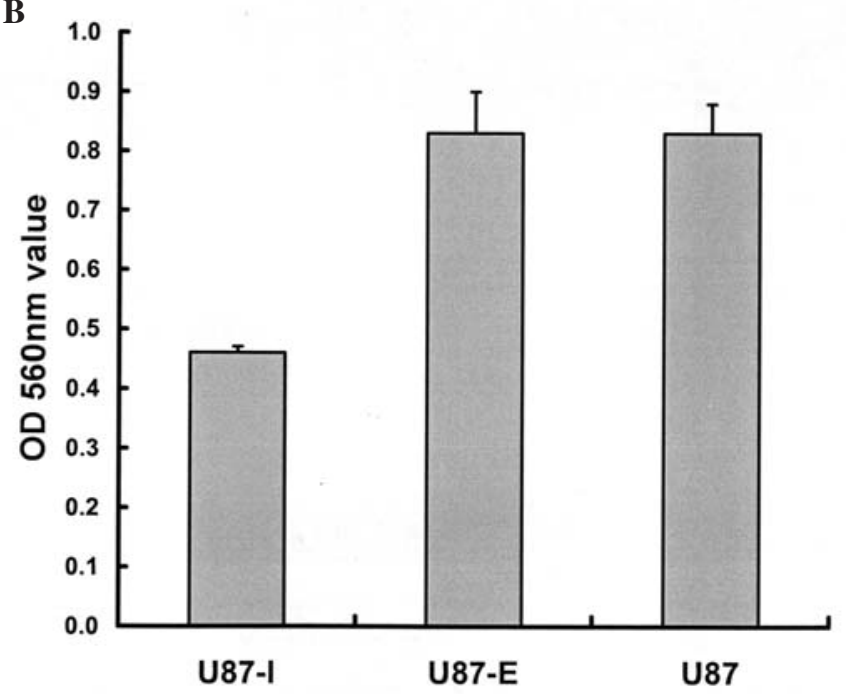

Figure 6. The effect of the hTERT-siRNA on cell invasive capacity in U87MG cells in vitro. (A) Microscopic views show a reduction in invading U87MG cells when cells infected with the hTERT-siRNA vector (magnification $\mathrm{x} 100$ ). The cells that had migrated through the transwell membrane were stained with Giemsa. (a), the hTERT-siRNA vector infected U87MG cells (U87-I); (b), the hTERT-NC vector infected U87MG cells (U87-E); (c), uninfected U87MG cells (U87). (B) Following the staining, the invaded cells were extracted and measured for optical density at $560 \mathrm{~nm}$. The OD560 values correlated positively with the number of cells migrating through the transwell membrane.

was specific to the delivery of hTERT-siRNA. Importantly, the in vivo glioma cell growth inhibition was already evident at day 6 post vector injection; the averages of tumor volume were $269.64 \pm 111.02 \mathrm{~mm}^{3}, 761.82 \pm 114.77 \mathrm{~mm}^{3}$ and $984.41 \pm 716.9 \mathrm{~mm}^{3}$ for the hTERT-siRNA vector, the hTERT- 


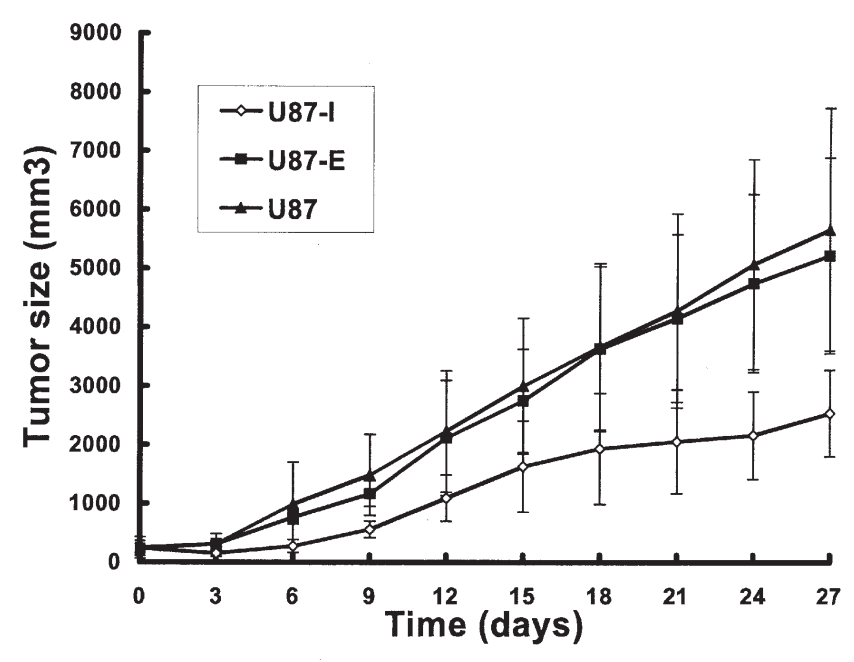

Figure 7. The antitumor effect of the hTERT-siRNA on the growth of U87MG glioma cells in vivo. A total of $1 \times 10^{7} \mathrm{U} 87 \mathrm{MG}$ glioma cells were transplanted subcutaneously into BALB/c-A nude mice. When the tumors were $5 \mathrm{~mm}$ in diameter, $5 \times 10^{7} \mathrm{TU}$ of either the hTERT-NC (U87-E), the hTERT-siRNA (U87-I) vector in $10 \mu 1 \mathrm{PBS}$, or $10 \mu \mathrm{l}$ PBS (U87) were injected into the tumor. The ability of the hTERT-siRNA to inhibit tumor growth in vivo was determined by measuring tumor volume every third day. Tumor size was calculated using the equation: $0.5 \mathrm{x}$ length $\mathrm{x}$ width ${ }^{2}$. All measurements were performed in a coded, blinded fashion.

$\mathrm{NC}$ vector and the PBS injected U87 group, respectively $(\mathrm{P}<0.05)$. The trend was maintained and was significant even at the end of the experiment at day 27 post vector injection.

\section{Discussion}

In the present study, we have engineered lentiviral vector encoding siRNA construct targeting hTERT mRNA. The lentiviral vectors were pseudotyped with VSV-G envelope and could mediate efficient gene delivery and maintain longterm transgene expression in normal cells from the central nervous system (17) and in glioma cells (12). Using U87MG cells as a model, we demonstrated that the hTERT mRNA and telomerase activity were significantly diminished following lentiviral vector mediated delivery of anti-hTERT siRNA. However, under our short-term in vitro culture conditions, diminished hTERT mRNA and telomerase activity showed no detectable effects on cell proliferation, cell cycle progression and telomere sequence shortening. In contrast, a profound reduction in the invasiveness capacity was observed as demonstrated in transwell migration assay. Lentiviral vector mediated delivery of anti-hTERT siRNA in vivo significantly inhibited the growth of pre-established U87MG cell xenograft glioma in immunodeficient mice. Importantly, our data strongly suggest that the inhibition of glioma cell invasion capacity and in vivo growth operated in a telomere length-independent manner.

Telomerase complexes, with its hTERT and hTR as essential components, have been suggested to be a key target for cancer treatment (18). In fact, clinical studies have shown that the presence of telomerase activity in glioma specimens may be a predictor of its malignancy potential (19-21) and the relative amount of hTERT mRNA may represent a diagnostic and prognostic indicator for GBM patients (9). Moreover, high level hTERT mRNA or high telomerase activity is associated with rapid tumor recurrence and/or poor therapeutic outcome in many other human tumors (22-24).

Previous studies have shown that downregulation of hTERT can lead to declined cell proliferation and/or induction of apoptotic cell death in glioma cells and other types of cancer cells (25-31). As human cells reduce their telomere length by 5-20 repeats during each cell cycle, there will be a lag phase between the onset of telomerase inhibition and the stage when cancer cell telomere sequences are sufficiently shortened to generate detrimental effects on chromosomal segregation during mitosis (18). Therefore, if the cell growth inhibition effect is telomere length-dependent, long-term suppression of telomerase expression will be required until cell growth inhibition could be evident (32). On the other hand, recent studies have suggested that hTERT contributes to tumorigenesis by a telomere length-independent mechanism (33). In line with this, siRNA mediated downregulation of RNA unit rapidly inhibited the cell growth and enhanced cell apoptosis in multiple types of cancer cells in a telomere length-independent manner $(34,35)$. In agreement with previously studies $(27,28)$, our data show that although hTERT mRNA and TRAP assay telomerase activity are diminished following lentiviral vector mediated delivery of anti-hTERT siRNA, the short-term in vitro growth capacity of such cells are not impaired. However, the in vivo glioma cell growth was significantly inhibited. But our data strongly suggest that the anti-hTERT siRNA mediated in vivo glioma cell growth inhibition effect is not due to telomere length shortening, because a significant growth inhibition was already detected at day 6 post lentiviral vector injection, a time period not sufficient for inducing telomere length shortening as assessed in in vitro infected glioma cells. Thus, anti-hTERT siRNA mediated in vitro and in vivo cancer cell growth inhibition can operate via different mechanisms.

Although remote metastasis is rare for glioma, intracranial infiltration of glioma cells represents a determining factor for the dismal treatment outcome. Our data also show that inhibition of hTERT can critically reduce the invasive capacity of glioma cells. Thus, inhibition of hTERT can reduce glioma cell proliferation as well as invasive capacities. Our findings are consistent with findings that the components of telomerase complexes are also involved in gene expression related to cancer cell proliferation and migration and also the angiogenesis process (35). To our knowledge, this is the first report demonstrating a direct involvement of hTERT in cell migration capacity. Further studies are required to clarify the mechanism for the finding of these studies.

In conclusion, we have demonstrated that lentiviral vector mediated stable expression of hTERT targeting siRNA can significantly inhibit the in vivo glioma cell growth in a telomere length-independent manner. Importantly, the inhibition of hTERT results in diminished invasive capacity in glioma cells. Thus, inhibition of hTERT may greatly improve the outcome of glioma treatment, and when used in combination with other measures may contribute towards a better clinical prognosis. 


\section{Acknowledgements}

This work was supported by Jiangsu Natural Science Foundation (JSNSF no. BK2004428). We thank Pengcheng Xun for assistance with data analysis. Xiaolong Fan is a $\mathrm{Li}$ Ka Shing Scholar. Dr Seema Rosqvist is thanked for critical reading of the manuscript.

\section{References}

1. De Angelis LM: Brain tumors. N Engl J Med 344: 114-123, 2001.

2. Schwartzbaum JA, Fisher JL, Aldape KD and Wrensch M: Epidemiology and molecular pathology of glioma. Nat Clin Pract Neurol 2: 494-503, 2006.

3. Cerni C: Telomeres, telomerase, and myc. An update. Mutat Res 462: 31-47, 2000.

4. Harada K, Arita K, Kurisu K and Tahara H: Telomerase activity and the expression of telomerase components in pituitary adenoma with malignant transformation. Surg Neurol 53: 267-274, 2000.

5. You Y, Pu P, Peng Q, Xia Z, Huang Q, Wang C and Wang G: Telomerase activity and regulation in human neuroepithelial tumors. Chin J Surg 40: 90-93, 2002.

6. Komata T, Kondo Y, Kanzawa T, et al: Treatment of malignant glioma cells with the transfer of constitutively active caspase-6 using the human telomerase catalytic subunit (human telomerase reverse transcriptase) gene promoter. Cancer Res 61: 5796-5802, 2001.

7. You Y, Pu P, Huang Q, et al: Antisense telomerase RNA inhibits the growth of human glioma cells in vitro and in vivo. Int J Oncol 28: 1225-1232, 2006.

8. Meyerson M, Counter CM, Eaton EN, et al: hEST2, the putative human telomerase catalytic subunit gene, is up-regulated in tumor cells and during immortalization. Cell 90: 785-795, 1997.

9. Tchirkov A, Rolhion C, Kemeny JL, et al: Clinical implications of quantitative real-time RT-PCR analysis of hTERT gene expression in human gliomas. Br J Cancer 88: 516-520, 2003.

10. Boldrini L, Pistolesi S, Gisfredi S, et al: Telomerase activity and hTERT mRNA expression in glial tumors. Int J Oncol 28: 1555-1560, 2006.

11. Wiznerowicz M and Trono D: Conditional suppression of cellular genes: lentivirus vector-mediated drug-inducible RNA interference. J Virol 77: 8957-8961, 2003.

12. Steffens S, Tebbets J, Kramm CM, Lindemann D, Flake A and Sena-Esteves M: Transduction of human glial and neuronal tumor cells with different lentivirus vector pseudotypes. J Neurooncol 70: 281-288, 2004.

13. Kanzawa T, Germano IM, Kondo Y, Ito H, Kyo S and Kondo S: Inhibition of telomerase activity in malignant glioma cells correlates with their sensitivity to temozolomide. Br J Cancer 89: 922-929, 2003.

14. Kim NW and Wu F: Advances in quantification and characterization of telomerase activity by the telomeric repeat amplification protocol (TRAP). Nucleic Acids Res 25: 2595-2597, 1997.

15. Hahnfeldt P, Panigrahy D, Folkman J and Hlatky L: Tumor development under angiogenic signaling: a dynamical theory of tumor growth, treatment response, and postvascular dormancy. Cancer Res 59: 4770-4775, 1997.

16. Martens UM, Brass V, Engelhardt M, et al: Measurement of telomere length in haematopoietic cells using in situ hybridization techniques. Biochem Soc Trans 28: 245-250, 2000.
17. Trono D: Lentiviral vectors: turning a deadly foe into a therapeutic agent. Gene Ther 7: 20-23, 2000.

18. Shay JW and Wright WE: Telomerase: a target for cancer therapeutics. Cancer Cell 2: 257-265, 2002.

19. Sano T, Asai A, Mishima K, Fujimaki T and Kirino T: Telomerase activity in 144 brain tumours. Br J Cancer 77: 1633-1637, 1998.

20. Le S, Zhu JJ, Anthony DC, Greider CW and Black PM: Telomerase activity in human gliomas. Neurosurgery 42: 1120-1124, 1998.

21. Sugita Y, Nakashima A, Kato S, Sakata K, Morimatsu M and Shigemori M: Telomerase activity in gliomas with the use of non-radioisotopic and semi-quantitative procedure for terminal repeat amplification protocol. Oncol Rep 7: 1087-1092, 2000.

22. Hiyama E, Yokoyama T, Tatsumoto N, et al: Telomerase activity in gastric cancer. Cancer Res 55: 3258-3262, 1995.

23. Clark GM, Osborne CK, Levitt D, Wu F and Kim NW: Telomerase activity and survival of patients with node-positive breast cancer. J Natl Cancer Inst 89: 1874-1881, 1997.

24. Taga S, Osaki T, Ohgami A, Imoto $\mathrm{H}$ and Yasumoto $\mathrm{K}$ : Prognostic impact of telomerase activity in non-small cell lung cancers. Ann Surg 230: 715-720, 1999.

25. Kraemer K, Fuessel S, Schmidt U, Kotzsch M, Schwenzer B, Wirth MP and Meye A: Antisense-mediated hTERT inhibition specifically reduces the growth of human bladder cancer cells. Clin Cancer Res 9: 3794-3800, 2003.

26. Zhang PH, Zou L and Tu ZG: RNAi-hTERT inhibition hepatocellular carcinoma cell proliferation via decreasing telomerase activity. J Surg Res 131: 143-149, 2006.

27. Pallini R, Sorrentino A, Pierconti F, et al: Telomerase inhibition by stable RNA interference impairs tumor growth and angiogenesis in glioblastoma xenografts. Int J Cancer 118: 2158-2167, 2006.

28. Falchetti ML, Fiorenzo P, Mongiardi MP, et al: Telomerase inhibition impairs tumor growth in glioblastoma xenografts. Neurol Res 28: 532-537, 2006.

29. Zhang Z, Yang X, Zhang Y, et al: Delivery of telomerase reverse transcriptase small interfering RNA in complex with positively charged single-walled carbon nanotubes suppresses tumor growth. Clin Cancer Res 12: 4933-4939, 2006.

30. Lin RX, Tuo CW, Lu QJ, Zhang W and Wang SQ: Inhibition of tumor growth and metastasis with antisense oligonucleotides (Cantide) targeting hTERT in an in situ human hepatocellular carcinoma model. Acta Pharmacol Sin 26: 762-768, 2005.

31. Kurvinen K, Syrjanen S and Johansson B: Long-term suppression of telomerase expression in HeLa cell clones, transfected with an expression vector carrying siRNA targeting hTERT mRNA. Int J Oncol 29: 279-288, 2006.

32. Hemann MT, Fridman JS, Zilfou JT, et al: An epi-allelic series of p53 hypomorphs created by stable RNAi produces distinct tumor phenotypes in vivo. Nat Genet 33: 396-400, 2003.

33. Stewart SA, Hahn WC, O'Connor BF, et al: Telomerase contributes to tumorigenesis by a telomere length-independent mechanism. Proc Natl Acad Sci USA 99: 12606-12611, 2002.

34. Li S, Rosenberg JE, Donjacour AA, Botchkina IL, Hom YK, Cunha GR and Blackburn EH: Inhibition of cancer cell growth induced by lentiviral delivery and expression of mutant-template telomerase RNA and anti-telomerase short-interfering RNA. Cancer Res 64: 4833-4840, 2004.

35. Li S, Crothers J, Haqq CM and Blackburn EH: Cellular and gene expression responses involved in the rapid growth inhibition of human cancer cells by RNA interference-mediated depletion of telomerase RNA. J Biol Chem 280: 23709-23717, 2005. 